\section{Intravitreal Bevacizumab for the Treatment of Choroidal Neovascu- larization after Eyeball Perforation during Retrobulbar Anesthesia}

\section{Dear Editor,}

Retrobulbar anesthesia is a common anesthetic procedure for ophthalmic surgery. Complications of the procedure ranging from the relatively commonly encountered retrobulbar hemorrhage to life-threatening respiratory arrest have been reported [1]. Ocular penetration/perforation can occur as a rare complication with a poor prognosis. Importantly, choroidal neovascularization (CNV) can occur as an unusual late complication of ocular penetration/ perforation during retrobulbar anesthesia [2]. Herein, we report a case that underwent intravitreal injection of bevacizumab for the treatment of macular $\mathrm{CNV}$ as a late sequela of retrobulbar anesthesia-related globe perforation.

A 53-year-old male patient was referred to our clinic with a diagnosis of globe penetration during retrobulbar anesthesia for cataract surgery in the left eye. He had diabetes, hypertension, and hyperlipidemia, but no ocular medical history other than cataract. The axial length was $25.55 \mathrm{~mm}$ in the right eye and $25.34 \mathrm{~mm}$ in the left eye. The uncorrected visual acuity was $20 / 16$ in the right eye using Snellen lines and hand motion in the left eye. Intraocular pressure was $13 \mathrm{mmHg}$ in the right eye and 20 $\mathrm{mmHg}$ in the left eye. Anterior segment examination revealed no abnormalities. Fundus examination revealed vitreous hemorrhage and two penetrating wounds in the left eye (Fig. 1A). The patient underwent pars plana vitrectomy, vitreous hemorrhage removal, endolaser, and gas $(18 \%$ SF6) tamponade. Two retinal holes were observed along the inferotemporal vascular arcade and approximately 2.5 disc diameters temporal to the fovea. Endolaser was performed around the retinal holes to prevent postoperative

Received: October 26, 2020 Final revision: November 12, 2020 Accepted: November 16, 2020 retinal detachment. On the fifth postoperative day, the visual acuity was $20 / 20$ in the left eye using Snellen lines. Intraocular pressure was $8 \mathrm{mmHg}$ in the left eye. After 2 weeks, fundus photography revealed retinal scarring without CNV (Fig. 1B). Thereafter, the patient was referred to a nearby hospital for regular checkup. After 1 year, the patient was referred again with decreased visual acuity in the left eye. The best-corrected visual acuity was 20 / 60 in the left eye. Fundus photography, optical coherence tomography, and fluorescein angiography revealed subfoveal CNV with subretinal fluid in the left eye (Fig. 1C-1E). For the treatment of $\mathrm{CNV}$, he received three intravitreal injections of bevacizumab (Avastin; Genentech, South San Francisco, CA, USA). After three monthly injections, the CNV was inactive without surrounding exudation and hemorrhage, and the visual acuity was maintained at $20 / 60$ in the left eye (Fig. 1F).

Although retrobulbar injections are generally regarded as safe, routine procedures, a wide variety of complications may occur. The incidence of ocular penetration/perforation during retrobulbar and peribulbar injection has been reported to be between $1 / 1,000$ and 1/4,000 [2]. Risk factors for globe penetration/perforation during retrobulbar or peribulbar anesthesia include axial myopia, the presence of a posterior or inferior staphyloma, multiple injections, a history of a previous scleral buckling procedure, and enophthalmos [3]. The patient in this case had no known risk factors other than a long axial length of $25.34 \mathrm{~mm}$ in the left eye.

There are a limited number of reports of CNV development secondary to choroidal ruptures occurring due to open eye injuries. However, there was only one case report of CNV development after ocular penetration/perforation that occurred during retrobulbar anesthesia [2]. In that case, intravitreal ranibizumab was administered as three monthly injections. The CNV had regressed at the 6-month follow-up after the injections. We have presented a rare case of pars plana vitrectomy for scleral penetration/perforation and vitreous hemorrhage that developed during retrobulbar anesthesia, followed by macular $\mathrm{CNV}$ occurrence after one year, which was treated using three intravitreal bevacizumab injections. After treatment, the CNV was inactive, but visual acuity did not improve. 

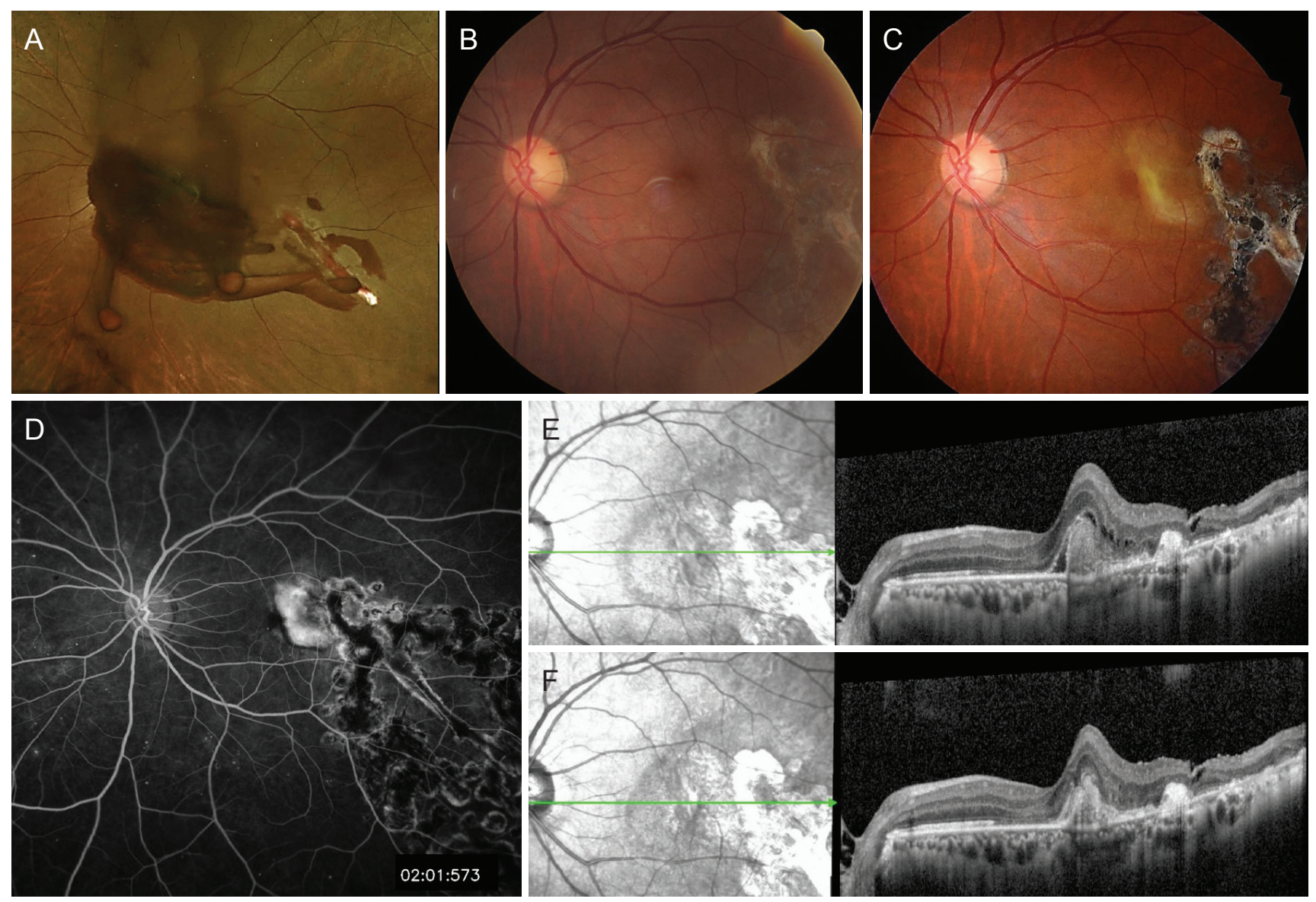

Fig. 1. Ophthalmic features (A) at initial visit, (B) after 2 weeks, and (C-F) after 1 year. (A) Preretinal hemorrhage and two penetrating wounds. (B) Retinal scarring without choroidal neovascularization was observed on fundus photography. (C-E) Choroidal neovascularization, subretinal fluid, and retinal scarring were observed on fundus photography, optical coherence tomography, and fluorescein angiography. (F) Choroidal neovascularization was inactive without surrounding exudation and hemorrhage after three monthly injections. Informed consent for publication of the clinical images was obtained from the patient.

To conclude, if globe penetration/perforation during local retrobulbar or peribulbar anesthesia occurs, we should keep in mind that $\mathrm{CNV}$ can develop as long as one year afterwards. If CNV develops after globe penetration/perforation during local retrobulbar or peribulbar anesthesia, anti-vascular endothelial growth factor treatment, such as bevacizumab, should be administered to prevent further visual decline. Further studies including a larger number of cases are needed to assess the long-term treatment outcomes and visual prognosis.

Kyung Won Kim, Se Joon Woo

Department of Ophthalmology, Seoul National University Bundang Hospital, Seoul National University College of Medicine, Seongnam, Korea

E-mail(SeJoonWoo): sejoon1@snu.ac.kr

\section{Conflict of Interest}

No potential conflict of interest relevant to this article was reported.

\section{Acknowledgements}

This work was partly supported by the National Research Foundation of Korea (NRF) grant funded by the Korea government (MSIT) (No. 2020R1F1A1072795).

\section{References}

1. Rosenblatt RM, May DR, Barsoumian K. Cardiopulmo- 
nary arrest after retrobular block. Am J Ophthalmol 1980;90:425-7.

2. Dikci S, Yilmaz T, Gok ZE, et al. Choroidal neovascularization secondary to ocular penetration during retrobulbar anesthesia and its treatment. Oman J Ophthalmol 2017;10:44-
6.

3. Duker JS, Belmont JB, Benson WE, et al. Inadvertent globe perforation during retrobulbar and peribulbar anesthesia. Patient characteristics, surgical management, and visual outcome. Ophthalmology 1991;98:519-26. 